\title{
Del estancamiento estabilizador al dinamismo productivo en México
}

\section{ISAAC SÁNCHEZ-JuÁREZ* Y ROSA M. GARCÍA-ALMADA**}

\section{RESUMEN}

Este artículo se enfoca en demostrar que la producción y empleo entre 2000-2017 registraron una evolución totalmente insatisfactoria como consecuencia del diseño y ejecución de una política económica centrada en la estabilidad de los precios y las finanzas públicas equilibradas. Para verificar lo anterior se utilizaron estadísticas oficiales. Entre los principales hallazgos se tiene que la economía creció de forma mediocre, en términos per cápita $0.94 \%$ en promedio y en materia de generación de empleo formal $1.02 \%$. Por lo tanto, se recomienda el establecimiento de un modelo de dinamismo productivo, con el ánimo de generar un cambio estructural positivo que eleve el crecimiento de la producción y empleo.

Palabras clave: Crecimiento económico, empleo, salarios, política económica.

Clasificación JEL: E60, E66, O11.

* Universidad Autónoma de Ciudad Juárez, Profesor de economía del Departamento de Ciencias Sociales Universidad Autónoma de Ciudad Juárez, Chihuahua, México. Correo electrónico: isaac.sanchez@uacj.mx

** Universidad Autónoma de Ciudad Juárez, Profesora de economía del Departamento de Ciencias Sociales Universidad Autónoma de Ciudad Juárez, Chihuahua, México. Correo electrónico: maria.garcia@uacj.mx 


\section{ABStract}

\section{From stabilizing stagnation to productive dynamism in Mexico}

This paper focuses on demonstrating that production and employment between 2000-2017 registered a totally unsatisfactory evolution as a result of the design and execution of an economic policy focused on price stability and balanced public finances. Official statistics were used to verify the above. The main findings are that during the period indicated the economy grew mediocrely, in terms per capita $0.94 \%$ on average and in the matter of formal employment generation $1.3 \%$. Therefore, it is recommended to establish a model of productive dynamism, with the aim of generating a positive structural change that increases the growth of production and employment.

Keywords: Economic growth, employment, salaries, economic policy. JEL Classification: E60, E66, O11.

\section{INTRODUCCIÓN}

El objetivo de este trabajo es simple: demostrar que entre 2000 y 2017 las principales variables macroeconómicas relacionadas con la economía real (producción y empleo) no evolucionaron favorablemente como consecuencia del diseño y ejecución de una política macroeconómica centrada en lo financiero, cuyos fundamentales son el control de la inflación y el mantenimiento de finanzas públicas equilibradas. Esto se ha traducido en una pérdida de bienestar para millones de mexicanos que ahora viven con algún grado de pobreza o vulnerabilidad (53,418,151 personas en 2016, según el Coneval), mientras existe un pequeño grupo que controla las instituciones, los cuales se han visto favorecidos y han incrementado su riqueza abonando a la profundización de la desigualdad económica.

La política económica lejos de contribuir al desarrollo se ha enfocado en crear condiciones de estabilidad para grupos financieros que, en la mayor parte de los casos, no viven en México, se trata de tomadores de decisiones extranjeros que modernizaron el viejo modelo de economía de enclave (extractivista), visualizado geográficamente como islas de prosperidad en medio de un mar de miseria y abandono (respecto a la financiarización de la economía, véase Levy, 2017).

El contexto actual es ideal para debatir si se quiere continuar con el actual modelo económico, conocido como Estancamiento Estabilizador 
(MEE) o si se crean condiciones para llevar a cabo un cambio estructural que conduzca a un nuevo modelo que, al menos en la parte macroeconómica, se concentre en el dinamismo de la producción (denominado MDP) con énfasis en la mejora de las condiciones laborales, particularmente en los salarios.

El pasado reciente ha sido decepcionante en este sentido, ya que, en el 2000, tras más de setenta años de un mismo partido en el poder presidencial (PRI), las personas democráticamente salieron a votar por un candidato de la derecha (PAN), el cual prometía grandes transformaciones, lamentablemente durante su gestión (2000-2006) y la siguiente (2006-2012) en materia económica se continuó con el MEE, por lo que en las elecciones de julio del 2012 la ciudadanía decidió que regresara al poder el partido hegemónico (PRI), el cual fiel a su tradición mantuvo con firmeza el MEE, con lo que se experimentó una transición política en México sin que ello se replicara en lo económico. Del 2000 al 2017, la política económica se configuró y ejecutó en la misma dirección.

Tras la victoria del candidato de la izquierda en las elecciones del 2018 se espera se transite del MEE al MDP, el cual deberá estar basado en el fortalecimiento de la producción y el empleo bajo la égida de una política fiscal y monetaria enfocadas a tal objetivo, que no descuida el control de los precios, para tener un enfoque dual (precios y crecimiento económico). Además de crear las condiciones para que se recupere la inversión pública, complemento de la inversión privada, y los salarios como parte de una estrategia de fortalecimiento del mercado interno.

El documento se estructuró en tres secciones. En la primera se presenta un poco de historia económica para comentar el origen, evolución y consecuencias del MEE. En la segunda se exponen los ciclos del crecimiento en México, que se caracterizan por su inestabilidad y profundidad en las recesiones, así como su sincronización con el ciclo norteamericano; también se presenta evidencia del estancamiento económico. En la tercera se muestra la evolución del empleo formal y los salarios. Se termina con algunas conclusiones de política económica para establecer un MDP.

\section{Modelo de estancamiento eStabilizador (MEE)}

Aunque el énfasis es en un periodo reciente, en realidad el origen del MEE se encuentra en los primeros años de los ochenta del siglo XX cuando 
se abandonaron las políticas de corte keynesiano por considerar que eran las causantes del quiebre en las finanzas públicas al ser demasiado populistas, en su lugar se comenzaron a establecer políticas que seguían las recomendaciones del Consenso de Washington: 1) disciplina fiscal mediante la reducción del gasto público; 2) privatización de empresas estatales; 3) liberalización comercial y financiera; 4) desregulación, 5) competencia económica y protección de los derechos de propiedad y 6) énfasis en el mercado como sustituto de la acción del Estado para la promoción del desarrollo económico (ver Williamson, 1998).

Se debe reconocer que estas medidas parecían bien intencionadas. Particularmente, Williamson (1998: 62) señala que el Consenso perseguía la creación de bancos centrales autónomos que evitarán malos manejos del circulante, causa en el pasado de inflaciones abrumadoras, así como comisiones presupuestarias fuertes, un poder judicial independiente e incorruptible y entidades promotoras de la productividad. De esta forma, como una imposición desde el exterior, ante un deterioro de los fundamentales de la economía y a manera de rescate se dio un giro en la concepción de la política económica.

Efectivamente, a principios de los ochenta la economía estaba sumida en el caos, con un sexenio de crecimiento cero (1982-1986) y un promedio de inflación del $70 \%$, con registros de incremento en el nivel general de precios superiores a los tres dígitos en algunos años. Adicional a esto, el proceso de reformas que se inicia en los ochenta se enmarca en la crisis de deuda, la cual obligó a la declaración de moratoria por tres meses, la nacionalización de los bancos y firmar acuerdos con el Fondo Monetario Internacional que hicieron obligatorio el establecer un programa de estabilidad y ajuste estructural (véase Huerta, 2009).

La transformación que sufrió México no fue exclusiva, formó parte de una cadena de cambios a nivel global, producto de una reestructuración del capitalismo que buscaba modernizarse para mantener los márgenes de ganancia de los grupos de poder internacionales. De esta forma se aplicaron medidas desde arriba hacia abajo por un grupo de especialistas técnicos, quienes, con un profundo desconocimiento de la sociedad y economía local (o bien omisión por no ser de importancia para ellos), deciden rediseñar las instituciones más importantes, como la Secretaría de Hacienda, de Economía (antes Secretaría de Comercio y Fomento Industrial) y el Banco de México para emular a sus similares 
en países desarrollados, particularmente los Estados Unidos de Norteamérica (véase Babb, 2003).

Respecto a la condicionalidad de las instituciones internacionales para instrumentar el MEE, Quintana (2014: 162-163), apunta lo siguiente:

Debemos acotar que la condicionalidad y el proceso de copia que ésta genera no son en modo alguno absolutos, ni son los únicos elementos que explican el proceso de reforma y el cambio institucional en México. Lo anterior significa que, en el diseño institucional, la condicionalidad debe ser considerada relativa. Esta última afirmación se refuerza si reconocemos que al interior del país y frente a la caída del mercado interno, los grupos oligopólicos se constituyeron en una presión efectiva para impulsar la apertura de la economía, pero sin contar con propuestas claras acerca de cómo se debía conducir dicho proceso. Es decir, los organismos internacionales influyeron en el rumbo de las reformas, pero el activismo del sector empresarial pro apertura económica les facilitó el camino.

Ahora bien, la apertura comercial que se inició en 1986 con la entrada al GATT, hoy Organización Mundial de Comercio (OMC), es el rasgo más importante del cambio de modelo económico, esto se fortaleció todavía más en 1994 con la entrada en operación del TLCAN con lo que se evitaba el regreso de políticas del pasado, especialmente la protección comercial y el establecimiento de una política de desarrollo industrial (ver Moreno-Brid et al., 2006).

Tras la estabilización económica en los ochenta y la reforma de los noventa, quedó definido el MEE, el cual se caracteriza por una política cambiaria flexible, una política comercial orientada a la eliminación de barreras comerciales y la promoción de nuevos tratados comerciales, una política monetaria conducida por un banco central autónomo que sigue el esquema de objetivos de inflación y una política fiscal basada en la austeridad, la cual tiene un comportamiento procíclico (véase Basilio, 2018). En la parte fiscal, el rasgo más preocupante es la reducción de la inversión pública como porcentaje respecto del PIB, de cerca de $10 \%$ en 1982 a casi $4 \%$ en 2015. A este respecto Moreno-Brid et al. (2016: 16), señalan:

... en las dinámicas competitivas de la globalización, tan centradas en la innovación y la productividad como factor competitivo por excelencia, contraer los cocientes de inversión invita al atraso económico. En otras palabras, la volatilidad de la inversión, la 
profundidad de fases recesivas y su dificultad en recobrar impulsos previos, construyen una dinámica perversa entre la fluctuación cíclica y la tendencia del crecimiento de largo plazo, que tarde o temprano erosiona el acervo de capital y con ello el crecimiento potencial. Este adverso fenómeno, junto con el patrón de debilitamiento de la inversión pública, ayuda a explicar la desaceleración de la economía nacional, si bien bajo las reformas de mercado, hay otros factores como el grado de desmadejamiento de la estructura productiva.

De esta forma, el MEE que se configuró se caracteriza en lo macroeconómico por su énfasis en la estabilidad y la obsesión con el control de los precios (lo que se justificaba a principios de los ochenta, pero no necesariamente ahora que la inflación es en promedio del $4 \%$ anual). Para lograr dicha estabilidad la principal estrategia consiste en mantener los salarios reducidos, contraer la demanda agregada y ralentizar la tasa de crecimiento económico ${ }^{1}$.

En lo productivo, el MEE no reconoce la importancia de una política industrial activa y salvo excepciones en los sectores automotriz, del software y aeronáutico, persiste la aplicación de políticas horizontales que dan un trato homogéneo a todos los sectores, sin reconocer que existen actividades industriales clave (ver Sánchez, 2013; Revilla et al., 2015 y Carbajal y de Jesús Almonte, 2017). De esta forma se ha configurado el MEE centrado en lo financiero y con una clara vocación hacia el exterior, lo que ha generado dependencia de la economía ${ }^{2}$.

Con lo expresado hasta ahora, conviene apuntar que el MEE tiene ingredientes que deben mantenerse, como la apertura comercial, pero ahora centrarse en la diversificación de mercados buscando una mayor conexión con Europa, China, y América Latina. También debería mantenerse la búsqueda de transparencia y fortalecimiento de las instituciones para el combate a la corrupción, lo mismo que la eliminación de trabas burocráticas a la creación de empresas y el fortalecimiento del sistema de ahorro y crédito para actividades productivas.

Por otra parte, en la actualidad, cinco problemas parecen persistir y por ello se propone el MDP: a). una baja tasa de crecimiento económico o estancamiento económico, b). ritmo lento de creación de empleo formal, c). bajos salarios, d). pobreza y e). desigualdad. Lo que sí se

Respecto al papel de los salarios como ancla de los precios, véase Perrotini y Vázquez (2017)..

Esto es particularmente claro en el caso de la sincronización entre el ciclo económico mexicano y americano, como lo demuestran Mejía et al. (2017). 
logró durante el MEE son déficits presupuestarios pequeños, baja inflación, incremento de las exportaciones no petroleras y aumento de la inversión extranjera directa, logros que no fueron suficientes para apuntalar el desarrollo económico mexicano ${ }^{3}$.

Sin embargo, para las autoridades (2012-2018) el éxito del MEE está por alcanzarse, basta con profundizar lo realizado, y por ello entre 2012 y 2013 propusieron e instrumentaron una serie de reformas de segunda generación (en la dirección propuesta por Martínez et al., 2004, para quienes la liberalización siempre es pertinente para generar crecimiento). Dichas reformas fueron las siguientes (entre paréntesis el año de arranque): 1) laboral (2012); 2) ley de amparo (2013); 3) educativa (2013); 4) hacendaria (2013); 5) financiera (2014); 6) transparencia (2014); 7) judicial (2014); 8) telecomunicaciones (2014); 9) energética (2014) y 10) competencia (2014).

Respecto a las reformas, en 1995 al año siguiente de que inicio el TLCAN, el PIB enfrentó una crisis severa reduciéndose 6.9\%; los años de 1996, 1997 y 1998 fueron buenos para el crecimiento, pero esto se terminó en los primeros años del siglo XXI, con lo que se dijo que era cuestión de esperar para ver el dinamismo productivo. En el 2018, tras cuatro años aproximadamente de aplicadas las primeras reformas se indicaba que se debía continuar esperando que los frutos tarde o temprano serán cosechados. Como se expone en el segundo apartado, de seguir con el MEEE lo único que se observará es desigualdad, pobreza, crisis periódicas, bajo crecimiento, precarización laboral y bajos salarios.

Antes de exponer los saldos negativos del MEE, conviene citar a la OCDE, organismo que celebraba que México seguía más del $70 \%$ de sus recomendaciones lo que se traduciría en mayores tasas de crecimiento:

En 2012, el gobierno electo alcanzó rápidamente un acuerdo histórico con partidos políticos de ideologías divergentes para establecer un ambicioso y consensuado paquete de reformas: el "Pacto por México", con el que se buscó volver a colocar al país en la senda de la prosperidad. Se realizaron trabajos legislativos para proponer medidas estructurales importantes que permitieran mejoras en materia de competencia, educación, energía, sector financiero, empleo,

3 Una lectura respecto al colapso económico como resultado de la aplicación del Consenso de Washington se encuentra en Yifu (2014). 
infraestructura, telecomunicaciones y sistema tributario, entre muchos otros ámbitos, cuya instrumentación ya dio inicio. Si estas reformas se implementan a plenitud, podrían aumentar la tendencia del crecimiento del pib per cápita anual en un punto porcentual durante los próximos diez años; los efectos de la reforma energética serán los más visibles en las etapas iniciales, mientras que los frutos de las reformas educativas se apreciarán, a más largo plazo, en los años venideros (OCDE, 2015:4).

La OCDE (2015), aseguraba que el crecimiento resultaría de las reformas, esto a pesar de un entorno internacional adverso caracterizado por la reducción de los precios del petróleo y el endurecimiento de la política monetaria norteamericana, incluso afirmaba en este reporte que se podría crecer un punto adicional si se llevaba a cabo una reforma en el sector agrícola para reducir las restricciones al uso de la tierra y eliminar los subsidios; es decir, deteriorando aún más las condiciones del sector real de la economía. En la figura 1 aparece la tasa de crecimiento promedio observada del producto por persona anual versus las estimaciones de la OCDE, de $0.6 \%$ en $1980-2013$ se pasaría a $2.6 \%$ entre 2014-2040.

\section{Figura 1}

CRECIMIENTO DEL PIB PER CÁPITA OBSERVAdo

Y PROYECTADO POR LA OCDE EN MÉXICO

(EN EL EJE VERTICAL DÓLARES PPC DEL 2005)

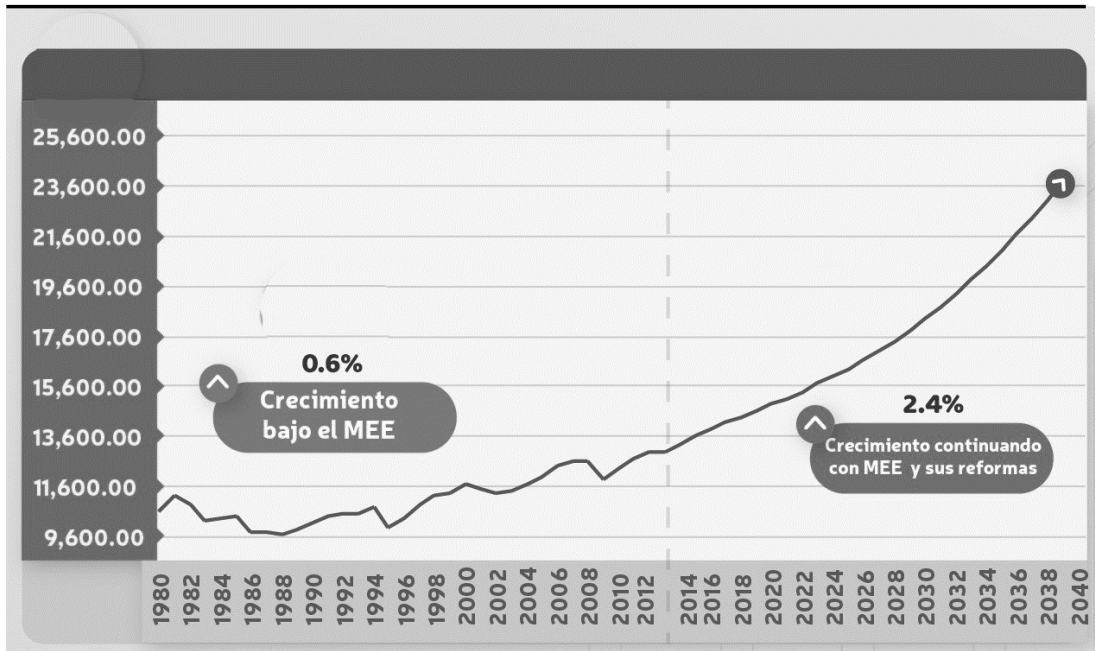

Fuente: Elaboración a partir de la OCDE (2015: 4). 
La insistencia en lo conveniente del modelo los llevó a declarar que "El gobierno ha emprendido grandes reformas para hacer más incluyente el crecimiento, disminuir la desigualdad del ingreso, mejorar la calidad de la educación, fomentar la formalidad y reducir la pobreza..." (OCDE, 2015: 4-5). Lo que al menos en materia de crecimiento, desigualdad y pobreza no es verdad y por ello la insistencia en generar un cambio estructural en la economía para tener un MDP $^{4}$.

Respecto a la desigualdad, Esquivel (2015: 7) ha calculado que el ingreso del $1 \%$ más rico en México es cerca de $21 \%$ del ingreso total y señala que el $10 \%$ más rico concentra el $64.4 \%$ de la riqueza nacional. Mientras el número de millonarios entre 2007-2012 creció un 32\%, en el mundo se redujo $0.3 \%$. La desigualdad es tan extrema, que se le puede poner nombre y apellido a los multimillonarios, entre los que sobresalen Carlos Slim, German Larrea, Alberto Bailleres y Ricardo Salinas (Esquivel, 2015: 8). La riqueza de los multimillonarios en 1996 era de 25,600 millones de dólares, mientras que en 2015 era de 142,900 millones de dólares. Esquivel (2015: 8) afirma que, en 2014, "los cuatro principales multimillonarios mexicanos podrían haber contratado hasta 3 millones de trabajadores mexicanos pagándoles el equivalente a un salario mínimo, sin perder un solo peso de su riqueza".

Esquivel (2016) señala que, si se considera la subestimación de los ingresos de la clase alta en las encuestas de ingresos y gastos de los hogares, entonces las mediciones oficiales de desigualdad indican que esta ha crecido, a diferencia de las cifras oficiales que muestran su reducción medida por el coeficiente de Gini (CG) sobre la base del ingreso corriente total. Las cifras oficiales indican que en 2000 el CG era 0.45 y 0.40 en 2012 , mientras que tras el ajuste se revela que era 0.60 en el primer año y 0.68 en el segundo ${ }^{5}$.

Entre las causas de esta desigualdad, Esquivel (2015) contempla la política fiscal, la cual según su opinión favorece a las personas de ingresos más altos, ya que no es progresiva, al gravar el consumo por encima del ingreso. Los más pobres al consumir pagan más que los

4 Para más detalle con relación a cómo las "nuevas" reformas no apuntan en la dirección deseada, se recomienda leer a Quintana (2014).

5 La desigualdad también se marca a nivel geográfico y sectorial, evidencia de ello se encuentra en García (2012). 
ricos. Los ricos también se benefician de la ausencia de un impuesto a las herencias y a las ganancias del capital.

Además de este factor causal de la desigualdad, Esquivel (2015) se concentra en la debilidad del mercado interno y en una política económica que asumía que el crecimiento económico cuando se presenta se transfiere de forma automática a todos los grupos de la sociedad, la cual también utilizó el control salarial como mecanismo de contención inflacionaria, lo que ha fortalecido la pobreza, ya que hoy en día una persona con un ingreso de un salario mínimo se considera como pobre extremo. Al respecto Campos y Monroy-Gómez-Franco (2016) encontraron que las ganancias en crecimiento económico no se han reflejado en reducciones sustantivas de la pobreza en México.

Con datos del Coneval (2016), la pobreza alimentaria (insuficiencia para obtener una canasta básica alimentaria, usando todo el ingreso disponible en el hogar), pasó de $24.1 \%$ de la población en 2000 a $19.6 \%$ en 2016, aparentemente se redujo, pero no es así, ya que en personas eran 23.7 millones en el primer año y 24 millones en el segundo. La población con un ingreso inferior a la línea de bienestar era de 54.7 millones de seres humanos en 2008 y se incrementó a 62 millones en 2016. Esto son los saldos de la política económica y social, las cifras refieren a personas cuyas vidas no son satisfactorias ${ }^{6}$.

México no ha podido, al igual que muchos países, incluyendo los desarrollados, reducir las desigualdades entre sus habitantes, lo que más allá de un modelo, parece ser la esencia misma del capitalismo. La pobreza, por otro lado, es un rasgo que se podría aminorar mediante un cambio de modelo económico que se concentre en la producción y en la creación de condiciones para ello. En las siguientes secciones se discuten algunas problemáticas adicionales: la frecuencia y volatilidad del ciclo económico (dinámica de la producción en el corto plazo), el estancamiento (dinámica de la producción de largo plazo), la baja tasa de generación de empleo formal, así como los magros salarios, todo ello como parte del MEE.

6 Respecto al fracaso de la política social, véase Sánchez-Juárez (2018). 


\section{CiClOS DEL CRECIMIENTO ECONÓMICO Y ESTANCAMIENTO}

\subsection{Los ciclos de crecimiento económico recientes en México}

Para identificar los ciclos del crecimiento en México se utilizó el filtro Hodrik-Prescott, el cual permitió descomponer la serie del PIB real a precios del 2013 en sus componentes tendencial y observado y a partir de aquí el ciclo. Dado que la serie utilizada correspondió al periodo 1993/01 a 2017/03, se detectaron tres ciclos completos. Para la referencia técnica sobre el cálculo de los ciclos, véase Sánchez-Juárez (2019).

Cuadro 1

CiCLOS ECONÓMICOS DE CRECIMIENTO EN MÉXICO, 1993/01 A 2017/03

\begin{tabular}{|c|c|c|c|c|c|c|c|c|c|}
\hline \multirow[b]{2}{*}{ \#Ciclo } & \multirow[b]{2}{*}{ Fondo } & \multicolumn{2}{|c|}{ Expansiones } & \multirow[b]{2}{*}{ Profundidad } & \multicolumn{4}{|c|}{ Recesiones } & \multirow[b]{2}{*}{ Volatilidad } \\
\hline & & Pico & Duración & & Pico & Fondo & Duración & Profundidad & \\
\hline & - & $1994 / 04$ & - & - & $1994 / 04$ & $1995 / 02$ & 9 meses & -11.56 & - \\
\hline 1 & $1995 / 02$ & $2000 / 03$ & $\begin{array}{l}5 \text { años y } \\
6 \text { meses }\end{array}$ & 39.71 & $2000 / 03$ & $2003 / 03$ & $\begin{array}{l}3 \text { años y } \\
3 \text { meses }\end{array}$ & -0.75 & 1.34 \\
\hline 2 & $2003 / 03$ & $2007 / 02$ & 4 años & 15.59 & $2007 / 02$ & $2009 / 02$ & $\begin{array}{l}2 \text { años y } \\
3 \text { meses }\end{array}$ & -8.23 & 1.29 \\
\hline 3 & $2009 / 02$ & $2012 / 02$ & $\begin{array}{l}3 \text { años y } \\
3 \text { meses }\end{array}$ & 15.48 & $2012 / 02$ & $2013 / 04$ & $\begin{array}{c}1 \text { año y } 9 \\
\text { meses }\end{array}$ & 1.44 & 0.94 \\
\hline & $2013 / 04$ & $2015 / 03$ & 2 años & 6.11 & $2015 / 03$ & - & - & - & - \\
\hline
\end{tabular}

Fuente: Elaboración propia.

El primer ciclo es el de mayor duración ( 8 años y nueve meses), la fase de expansión fue la más larga y la de mayor profundidad de los tres, lo que se corresponde con los primeros años de operación del TLCAN(tras la superación de la crisis del 94-95 y la recesión más profunda de la historia reciente de México). El primer ciclo también registró la recesión más larga, pero no la más profunda. Además, el primer ciclo fue el más volátil; es decir, los valores del PIB cíclico se alejaron de su media, por lo que el crecimiento trimestral y anual fue irregular-reducido.

Durante este primer ciclo la causa de la recesión 2000/03-2003/03 fue la crisis del mercado financiero en los EE.UU, la que se transmitió a México por medio de la sincronización de los ciclos industriales. Desde 
la década de los noventa por el sobrecalentamiento de la economía de los EE.UU se produjo un persistente déficit en cuenta corriente, el cual a su vez produjo una burbuja tecnológica que colaboró en la crisis; otro elemento que detonó el problema fue el crecimiento de la posición deudora del sector privado, que invertía a tasas elevadas en sectores tecnológicos y de telecomunicaciones, lo que hizo subir sus valores para finalmente desplomarse ante dos eventos no esperados: el ingreso de China a la OMC y los atentados terroristas del 11 de septiembre del 2001.

El segundo ciclo duró 6 años y tres meses, de los tres ciclos identificados en el cuadro 1, en éste la fase recesiva fue la más profunda, pero todavía lejos de la observada entre 1994-1995; este segundo ciclo fue tan volátil como el primero. Por otro lado, la etapa expansiva fue considerable y profunda, de hecho, los datos indican que para los tres ciclos el tiempo de duración de las recesiones es menor que el de las expansiones; sin embargo, las caídas críticas que se presentaron en 1995, 2001 y 2008 fueron suficientes como para alejar el PIB observado de su tendencia y con ello deteriorar las condiciones de bienestar macroeconómico.

El tercer ciclo se caracteriza por su poca duración, apenas cinco años, sobresale porque la profundidad de su etapa expansiva es similar a la del segundo ciclo y también porque durante su corta etapa recesiva el PIB entre el pico y el fondo creció $1.44 \%$, a diferencia de los otros dos ciclos en los cuales se contrajo la producción. Resumiendo, este tercer ciclo es el menos volátil de todos, es de corta duración y muy poca profundidad en la recesión, no así en la expansión.

Respecto a las causas de la recesión del 2008 destacan: 1) una política monetaria relajada con tasas de interés muy reducidas; 2) la creación de una burbuja inmobiliaria a partir de los noventa por el auge de la compra de vivienda y 3) un sistema financiero sin regulación prudencial, que prestó recursos a personas sin capacidad de pago. Esta crisis marca el inicio de una nueva era en la economía mundial al colapsarse el sistema financiero del centro hegemónico y revelar la fragilidad de las economías que se pensaba estaban desarrolladas. Los mecanismos de transmisión hacia México fueron la inversión extranjera directa, las exportaciones y las remesas (Mejía et al., 2017).

Las recesiones del 2000-2003 y 2007-2009 mostraron la fuerte y perniciosa sincronización que se tiene con la economía de los EE.UU, 
evidenciaron que no obstante el tener fundamentos macroeconómicos "sólidos", no hay blindaje ante los choques externos en una economía abierta, por lo que es de esperarse que éstos sigan determinando la aparición de nuevas recesiones en el futuro (Mejía et al., 2017).

De particular importancia es que en 2013/04-2015/03 se identificó la etapa expansiva de un nuevo ciclo, precisamente en 2015/03 alcanza su pico, por lo que la economía ha comenzado a desacelerarse y todo parece indicar que entre el 2018-2020 se registrará un nuevo fondo y con ello se confirmaría la etapa recesiva de este último ciclo (ver cuadro 1). El cambio de gobierno y la ratificación o no del T-MEC sin duda serán factores que presionarán para sufrir esta nueva recesión.

Ante este escenario, Mejía et al. (2017: 79), concluyen lo siguiente:

Los hechos son claros: las fases del ciclo estadounidense se reflejan inmediatamente en la economía nacional, o para decirlo de otro modo, la dinámica de la última depende de la primera, con muy poco margen de maniobra para la política de estabilización. Por tanto, ante las limitaciones que enfrenta para mitigar los choques de corto plazo, la preocupación central de la política tendría que ser el crecimiento de largo plazo.

\subsection{El estancamiento económico de largo plazo de la economía mexicana}

Se pasa ahora a exponer el estancamiento económico que prevalece, para lo cual primero se considera la siguiente regla: una economía experimenta una situación de crecimiento acelerado cuando el PIB per cápita supera el $2 \%$ por lo menos durante un periodo de diez años. Por lo que si una economía crece por debajo de $2 \%$ puede decirse que se encuentra estancada (Calderón-Villarreal y Hernández-Bielma, 2016: 177).

Entre 1980 y 1989 el decrecimiento per cápita fue $0.03 \%$, de 1990 a 1993 creció $1.99 \%, 1.75 \%$ de 1994 a 1999 y $0.94 \%$ del 2000 al 2017 , con lo que la economía mexicana se encuentra estancada desde hace treinta y ocho años (The Conference Board, 2018). Salvo los primeros años de la década de los noventa, el crecimiento ha sido bastante mediocre, aún más si se le compara con lo logrado en un pasado un poco más lejano, los últimos momentos del modelo desarrollista, entre 1970 y 1980 cuando la economía creció $3.36 \%$. El crecimiento económico en México se caracteriza por su volatilidad, insuficiencia y desigualdad (ver gráfica 2). 
Gráfica 2

LOGARITMOS Y CRECIMIENTO DEL PIB PER CÁPITA EN MÉXICO, 1951-2017

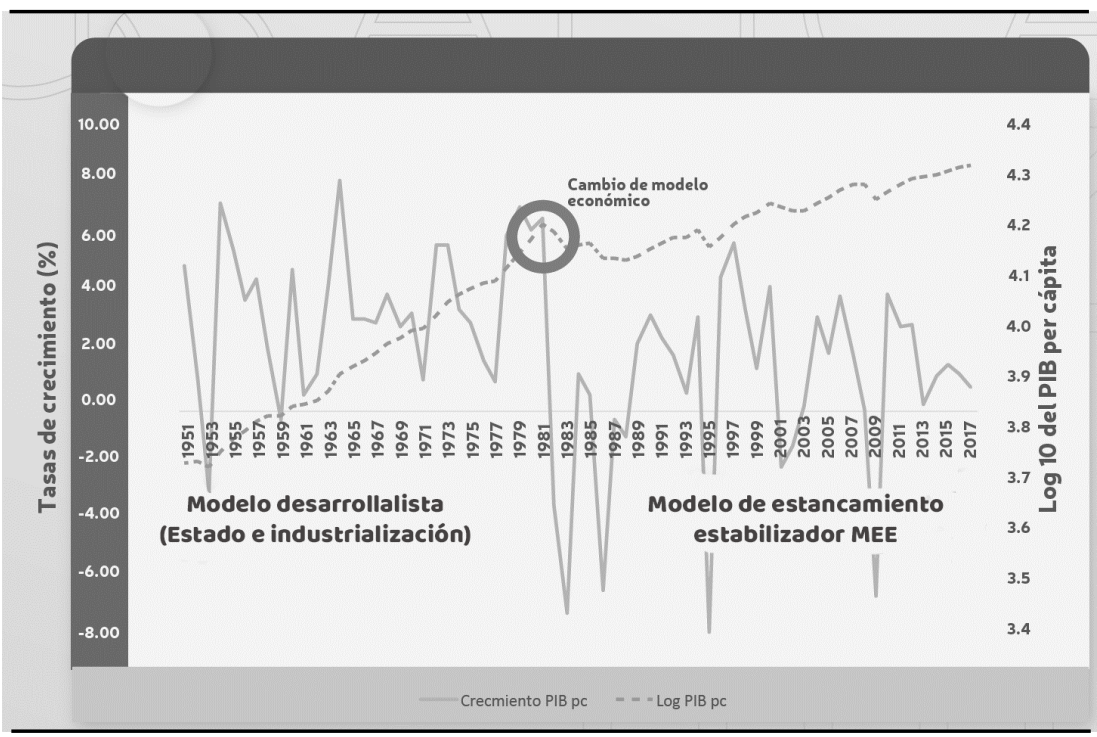

Fuente: Elaboración propia a partir de The Conference Board (2018).

El crecimiento promedio de 1982 a 2000, época en la que estuvo al frente el PRI, fue de $0.53 \%$; mientras que del 2001-2006, ya con un gobierno del Pan, fue $1.0 \%$ y $0.87 \%$ del $2007-2012$. Recientemente, con el regreso del PRI, entre 2013-2017 el crecimiento promedio per cápita fue $0.97 \%$, con lo que se concluye que la economía ha estado estancada. Algo que llama la atención es que entre 1951-1981 la economía creció $3.45 \%$, gobernada por el PRI, pero bajo la égida de un modelo centrado en la inversión pública y la industrialización con un fuerte énfasis nacionalista. Después dicho partido y su cúpula fue controlada por un grupo de economistas técnicos que impusieron el MEE con los resultados que se han expuesto. Lo que deja la lección de que no necesariamente mejorar las condiciones económicas supone un cambio de partido en el poder sino de la forma en la que quienes toman el poder conciben el desarrollo económico nacional y los intereses a los cuales responden.

¿Qué explica el estancamiento en México? Existen muchas respuestas, pero varios autores coinciden en el fracaso de la política económica durante el mee e indican que es necesario dinamizar la inversión productiva, particularmente la pública. También recomiendan 
eliminar la dependencia con los EUA, evitar la apreciación del tipo de cambio real y modificar la política monetaria para que no contribuya a la atonía de la inversión, así como instrumentar políticas industriales activas (Ibarra, 2008; Ros, 2015; Srithongrung y Sánchez-Juárez, 2015; Sánchez-Juárez y García-Almada, 2016; Moreno-Brid et al., 2016; Sánchez y Moreno-Brid, 2016 y Trejo, 2017)

Respecto a la necesidad de modificar la política monetaria, Levy (2014) demuestra que el Banco de México, al asumir de manera explícita la tasa de interés como instrumento y adoptar el control de la inflación como único objetivo, provoca que las variaciones de la tasa de interés del banco no amplíen la demanda, ni acercan la demanda efectiva al producto potencial y por tanto no se genera crecimiento económico. En Loría (2016) se presentan evidencias respecto a la forma en la que la política cambiaria antiinflacionaria es una de las restricciones al crecimiento; para probarlo, el autor usó un modelo SVAR con datos del periodo 1950-2014, el cual ayuda a demostrar que las recurrentes apreciaciones cambiarias han afectado los principales equilibrios macroeconómicos y se han convertido en una buena explicación del estancamiento.

Ahora bien, Vargas y Luna (2014), en un artículo sustentado en la teoría kaleckiana y post keynesiana, para el caso mexicano concluyen que la principal causa del estancamiento es la concentración del ingreso lo que se ha fortalecido por la aplicación de política neoliberales que han favorecido las ganancias corporativas y los ingresos de la clase alta, descuidando el mercado interno y las políticas de bienestar social. Por lo que concluyen que debe ampliarse el mercado interno por la vía de mejoras en las condiciones salariales.

Tras conocer los ciclos y el estancamiento económico de largo plazo en México, en la siguiente sección se pone en evidencia que existe un fuerte rezago en materia de creación de empleo formal y que los salarios no han crecido al ritmo requerido para contribuir a una mejora del bienestar social por lo que se fortalece la trampa del subdesarrollo.

\section{MERCADO LABORAL: BAJO RITMO DE GENERACIÓN DE EMPLEO Y SALARIOS}

Sobre la base del crecimiento de la PEA se sabe que en México se ha pasado de una necesidad de cerca de 700 mil empleos formales a inicios 
del TLCAN a aproximadamente 1 millón 200 mil empleos en 2017, que al no generarse profundiza la frustración y desesperación económica, particularmente entre los más jóvenes. El crecimiento promedio anual del empleo formal fue $4.2 \%$ entre 1970 y $1982,2.0 \%$ de 1982 a 1993 , $1.3 \%$ de 1994 al 2017 y $1.02 \%$ del 2000 al 2017.

De 1994 al 2000 se crearon en promedio anual 376,560 empleos formales, al final durante este periodo solamente se crearon 2,635,921 empleos, en 1995 se registró una crisis en el empleo al perderse 366,531 plazas. Del 2001 al 2006 se crearon en promedio anual 152,592 empleos y el periodo sumó 915,557 empleos; en 2001se registró una crisis de empleo al perderse 196,985 empleos. Del 2007 al 2013 se crearon en promedio anual 298,198 empleos y el periodo sumó 2,087,388 empleos; en 2008-2009 se perdieron 314,597 plazas. En el periodo más reciente, 2014-2017, en promedio anual se generaron 593,531 empleos con lo que es el periodo que más empleos ha sumado: 1,780,595 en cuatro años; no obstante, el déficit acumulado en estos veinticuatro años sigue siendo elevado.

La gravedad del problema del mercado laboral en México puede sintetizarse a partir de la tasa de informalidad que en enero de 2005 era $59.1 \%$ de la población ocupada total y de $56.3 \%$ en diciembre del 2017. La tasa más alta se registró en octubre del 2009 con $61.1 \%$, lo que revela que las caídas en la producción se correlacionan con pérdida de empleo y/o empeoramiento de las condiciones laborales, de ahí la necesidad de alentar el crecimiento económico sostenido y que sus beneficios alcancen a una mayoría; es decir, que sea lo menos desigual.

EL TLCAN contempló y se enfocó en la movilidad de bienes y servicios y por supuesto capital, pero excluyó la libre movilidad de personas, lo que probablemente hubiera impulsado la convergencia económica entre los tres países y constituido una auténtica región económica. De acuerdo con datos del Buró de Censos de los Estados Unidos de Norteamérica, en 1994 había 6,985,253 mexicanos nacidos en México residentes en aquel país, dicha cifra se incrementó paulatinamente hasta alcanzar un máximo de 12,211,129 en 2015, un crecimiento promedio anual de $3.05 \%$. En 2017, la cifra se estima en 12,006,942 mexicanos que viven en Norteamérica, muchos de los cuáles tiene una permanencia ilegal.

Los que dejan México lo hacen porque no encuentran condiciones apropiadas en el mercado laboral local, convirtiendo la migración en 
válvula de escape, y a medida que pasa el tiempo, ha perdido de importancia ante el endurecimiento de los controles de acceso y en general el empeoramiento de las condiciones económicas en el país vecino. De un máximo de 855,682 migrantes con destino a EE.UU. en 2007, la cifra cayó drásticamente hasta 96,136 seres humanos en 2015, último dato reportado por El Colegio de la Frontera Norte a través de su encuesta sobre migraciones.

La precarización del mercado de trabajo es otro elemento que se suma a la baja tasa de generación de empleos formales. Al respecto conviene citar a Pérez y Ceballos (2019: 128):

En general, los resultados econométricos señalan que, en una década, la clase subordinada y remunerada ha incrementado su probabilidad de experimentar mayor precariedad laboral, sin importar el sector, el sexo, la educación, el tamaño del establecimiento, la edad o el estado civil... la precariedad ha sido producto de las decisiones de las empresas que, en su búsqueda por obtener mejores beneficios, han reducido los costos de producción, a través de reducir salarios y prestaciones y aumentar jornadas de trabajo en la clase trabajadora asalariada que, en México, se compone por 68 por ciento del total de la población que labora.

Por si el bajo crecimiento de la producción y el empleo no fueran problemas suficientes, los salarios que recibe la clase trabajadora han empeorado, con lo cual se ha debilitado sensiblemente el mercado interno, lo que impide crear un nucleó endógeno de causación acumulativa. Los bajos salarios han favorecido la atracción de inversión extranjera directa y al igual que sucede en muchos países en vías de desarrollo contribuyen a crear mercancías de bajo precio que se venden en todo el mundo, particularmente en países desarrollados. Por cierto, la estrategia de salarios bajos no es exclusiva de México, forma parte de la nueva división internacional del trabajo y de la forma en la que opera el capitalismo desigual que favorece al capital y los capitalistas.

Con cifras proporcionadas por el Coneval, en su portal en línea, se sabe que en 1994 el ingreso mensual por persona era de 3,322 pesos; en 1996 bajó a 2,200 pesos; en 2002 fue de 3,100 pesos; en 2006 fue 3,350 pesos el máximo alcanzado durante la era TLCAN; en 2010 2,900 pesos y en 2014 3,015 pesos reales, con lo que es menor al existente en 1994, lo que revela el deterioro sufrido. Con un ingreso tan bajo, el acceso a 
Gráfica 3

SALARIOS MÍNIMOS GENERALES, ÍNDICE REAL, 2012=100

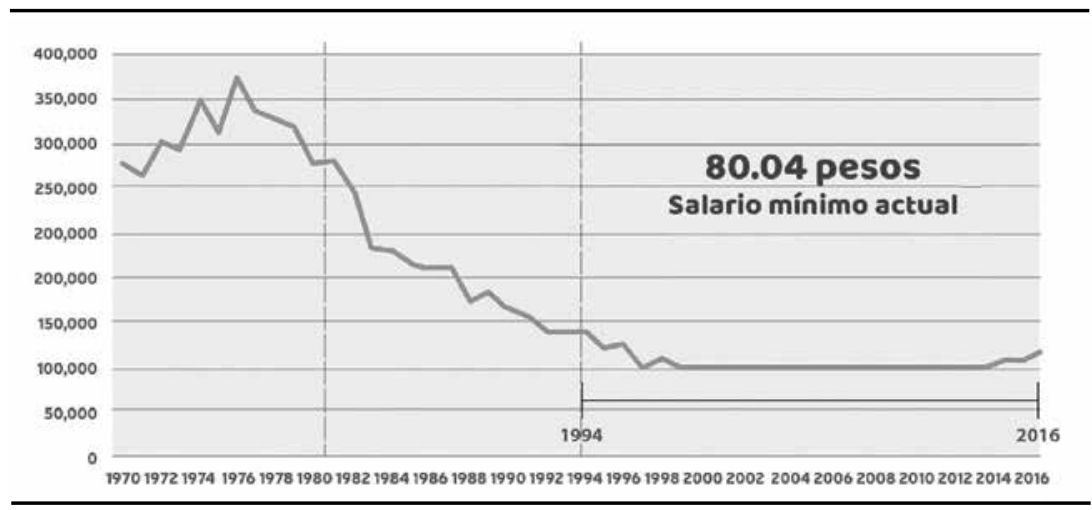

* El dato de salario mínimo actual corresponde a 2017.

Fuente: Elaboración propia con datos del Banco de México

satisfactores esenciales como educación, salud, alimentación, vestido y vivienda es complicado, el ingreso promedio es de franca subsistencia. En la gráfica 3 se muestra el deterioro que han sufrido los salarios mínimos reales, ya que de 1970 a 1981 el índice era en promedio de 319, mientras que de 1982 a 1993 fue 174 y del 2000 al 2017 de 101, en este periodo incluso se puede observar un notable estancamiento que se explica porque el salario mínimo funciona como mecanismo de control de la demanda agregada y estabilización de otros precios en la economía, como el principal objetivo de la actual política económica (para más detalle véase Perrotini y Vázquez, 2017).

\section{Conclusiones}

Existen dos instituciones primordiales en el diseño y ejecución de la política económica mexicana: La Secretaría de Hacienda y Crédito Público y el Banco de México, entre las dos configuran las políticas fiscal, monetaria, cambiaria y comercial. Al tener ahora un gobierno federal emanado de la izquierda existe la posibilidad de alterar su concepción, trasfondo ideológico y técnico para generar un cambio estructural y transitar de un modelo que privilegia lo financiero y la estabilidad a uno que busque fomentar lo real-productivo con énfasis en aquellas personas que hoy viven en situación de miseria. Se debe crecer 
a tasas elevadas y sostenidas para generar empleos de calidad caracterizados por salarios altos y prestaciones conforme al marco legal.

La tarea no es simple ya que se sabe que en el mundo globalizado actual existen condicionamientos que se imponen por parte de agentes económicos con gran fortaleza en países desarrollados; que, al gobierno local y constitucional, en los hechos, se le suma el "gobierno" internacional, lo que ha frenado todo intento de revolución económica no solo en México sino en América Latina.

También se debe evitar caer en la inocencia y reconocer que existe una parte de la política fiscal que está determinada por el Congreso de la Unión y por tanto, a pesar de que exista un ejecutivo comprometido con las ideas que a continuación se resumen, enfrentará dificultades para realizar los cambios necesarios, ya que existe un grupo no cuantificado, pero que se sabe numeroso y poderoso de cabilderos que susurran a los oídos de los congresistas para dejar las cosas en el camino marcado por el MEE.

Como se ha tratado de mostrar en el documento, el MEE no debe continuarse porque atenta contra variables fundamentales del bienestar humano como son el crecimiento económico, empleo y salarios. De mantenerse se seguirá ampliando la brecha que separa a los escasos ricos con los numerosos pobres. Lo que se propone aquí es pasar a un MDP que fomente el mercado interno, continúe con la estabilización de precios, fortalezca la liberalización comercial y sobre todo, privilegie el crecimiento económico y la generación de empleo formal. Para esto, habrá que establecer políticas fiscales y monetarias contra cíclicas, así como evitar apreciaciones del tipo de cambio que deterioran la competitividad. Se requiere retomar la visión desarrollista del pasado, actualizada bajo las nuevas condiciones del contexto internacional y el reconocimiento de los errores previos. De particular importancia en la agenda es la reducción de la sincronización del ciclo económico mexicano con el norteamericano, como se expuso están demasiado enlazados, particularmente en coyunturas recesivas, para lograr esto debe diversificarse el comercio internacional y fomentar la creación y mantenimiento de grandes empresas nacionales en sectores estratégicos, lo que hace urgente implementar una política industrial activa.

Se anticipa que tras dos años de desaceleración, otro de recuperación, en el cuarto se comenzará a trazar un proyecto de nación de 
largo plazo, que en lo económico debe caracterizarse por las siguientes condiciones: 1) Una política fiscal que se aboca al dinamismo productivo, fortalecimiento de la demanda y creación de nuevos empleos, centrada en la parte impositiva en obtener financiamiento por medio de gravar el ingreso más que el consumo, un impuesto a las herencias y a la entrada-salida de capitales; 2) política monetaria administrada por un banco central con doble mandato, fomentar el crecimiento y estabilizar los precios; 3) política cambiaria que evita la apreciación de la moneda, para lograr esto, se debe mantener un menor nivel de precios internos respecto a los externos, además las autoridades necesitan enfocarse en modernizar y elevar la productividad de la industria nacional; 4) política comercial enfocada en Europa, Asia y América Latina, ante la disminución del comercio con los EUA, lo que ayudaría a reducir la dependencia que hoy se tiene; 5) una política industrial activa, con liderazgo del Estado, centrada en la creación de infraestructura pública y financiamiento a la creación de grandes campeones nacionales, así como promoción auténtica de micro y pequeñas empresas: 6) reforma al sector energético para realmente reducir el precio de los insumos, tales como gasolina, gas y electricidad; 7) incremento al salario mínimo general en 51\% (dicha propuesta se toma de Campos, 2015), 8) revisión de la actual reforma educativa y el resto de reformas implementadas desde 2014 para que sean congruentes con el MDP propuesto, 9) fomento a la inversión en ciencia y tecnología para alcanzar $1 \%$ del PIB y 10) políticas con enfoque regional que atienden las necesidades y condiciones específicas de la diversidad geográfica que prevalece en México.

El reloj sigue su marcha inexorable, postergar por más años el cambio estructural centrado en el dinamismo productivo y las personas menos favorecidas puede sin temor a equivocación condenar al atraso permanente aquellos que hoy sobreviven con las migajas que derraman el pequeño grupo de privilegiados, para los que México es un país fuerte, dinámico y justo, cada vez más parecido a su verdadero amor: la economía de los Estados Unidos de Norteamérica. 


\section{BIBLIOGRAFÍA}

Babb, Sara (2003), Proyecto México: los economistas del nacionalismo al neoliberalismo, Fondo de Cultura Económica, Ciudad de México.

Basilio, Eufemia (2018), "Política fiscal procíclica y estabilidad monetaria en Brasil, Chile, Colombia, México y Perú”, Problemas del Desarrollo, 49(192), Instituto de Investigaciones Económicas UNAM, México, pp. 139-167.

Calderón-Villarreal, Cuauhtémoc y Leticia Hernández-Bielma (2016), “Cambio estructural y desindustrialización en México", Panorama Económico, 12(23), Escuela Superior de Economía, Instituto Politécnico Nacional, México, pp. 153-190.

Campos, Raymundo (2015), “El salario mínimo y el empleo. Evidencia internacional y posibles impactos para el caso mexicano", EconomíaUNAM, 12(36), Facultad de Economía UNAM, México, pp. 90-106.

Campos, Raymundo y Luis Monroy-Gómez-Franco (2016), “La relación entre crecimiento económico y pobreza en México", Investigación Económica, 75(298), Facultad de Economía UNAM, México, pp. 77-113. DOI: https:// doi.org/10.1016/j.inveco.2016.11.003

Carbajal, Yolanda y Leobardo de Jesús Almonte (2017), "Manufacturing labor in the central region of Mexico. An estimation by great división", Contaduría y Administración, 62(3), Facultad de Contaduría UNAM, México, pp. 902-923. DOI: https://doi.org/10.1016/j.cya.2017.05.004

Coneval (2016), Evolución de las dimensiones de pobreza, 1992-2016. Recuperado de https://coneval.org.mx/Medicion/Paginas/Evolucion-de-lasdimensiones-de-pobreza.aspx

Esquivel, Gerardo (2015), Desigualdad extrema en México, Oxfam, Ciudad de México.

Esquivel, Gerardo (2016), "Pobreza y desigualdad. Debates críticos sobre su medición e importancia", XXIX Congreso Nacional de Estudiantes de Economía, Tepic, Nayarit, México.

García, Rosa (2012), Liberalización comercial, descentralización territorial y polarización económica en México, Universidad Autónoma de Ciudad Juárez, Ciudad Juárez.

Huerta, Arturo (2009), "La liberalización económica y la estabilidad macroeconómica", EconomiaUNAM, 6(18), Facultad de Economía de la UNAM, México, pp. 89-105.

Ibarra, Carlos (2008), "La paradoja del crecimiento lento de México", Revista de la Cepal, 95, Cepal, Santiago de Chile, pp. 83-102.

Levy, Noemi (2014), "La política monetaria y el crecimiento económico: la tasa de interés de referencia del Banco de México", Economíalnforma, 387, Facultad de Economía UNAM, México, pp. 21-42. 
Levy, Noemi (2017), "Financiarización y modelo de acumulación: La evolución de las deudas y el sector externo de la economía mexicana", Análisis Económico, 32(79), Universidad Autónoma Metropolitana, México, pp. 53-75.

Loría, Eduardo (2016), "México: Crecimiento económico restringido y tipo de cambio, 1950-2014", Problemas del Desarrollo, 47(186), Instituto de Investigaciones Económicas UNAM, México, pp. 133-160.

Martínez, Lorenza, Aarón Tornell y Frank Westerman (2004), "Globalización, crecimiento y crisis financieras. Lecciones de México y del mundo en desarrollo", El Trimestre Económico, 71 (282), Fondo de Cultura Económica, México, pp. 251-351.

Mejía, Pablo, Miguel Díaz y Reyna Vergara (2017), "Recesiones de México en los albores del siglo XXI", Problemas del Desarrollo, 48(189), Instituto de Investigaciones Económicas UNAM, México, pp. 57-84.

Moreno-Brid, Juan, Jesús Santamaría y Juan Rivas (2006), "Manufactura y TLCAN: un camino de luces y sombras", EconomíaUNAM, 3(8), Facultad de Economía UNAM, México, pp. 95-114.

Moreno-Brid, Juan, Jamel Kevin e Israel Valverde (2016), Tendencias y ciclos de la formación de capital fijo y la actividad productiva en la economía mexicana, 1960-2015. Cepal, Santiago de Chile.

OCDE (2015), Estudios económicos de la OCDE: México 2015. OCDE, Paris.

Pérez, Jorge e Isabel Ceballos (2019), "Dimensionando la precariedad laboral en México de 2005 a 2015, a través del modelo logístico ordinal", Nóesis. Revista de Ciencias Sociales y Humanidades, 28(55), Universidad Autónoma de Ciudad Juárez, México, pp. 109-135. DOI: http://dx.doi. org/10.20983/noesis.2019.1.6

Perrotini, Ignacio y Alberto Vázquez (2017), "Is the wage rate the real anchor of the inflation targeting monetary policy framework?", Investigación Económica, 76(302), Facultad de Economía UNAM, México, pp. 9-54.

Quintana, Luis (2014), "Reforma económica y desempeño regional en México", en Jesús Montes de Oca (comp.), Nueva reforma del Estado. Los retos en México 2013-2018. Universidad Nacional Autónoma de México, Ciudad de México, pp. 157-180.

Revilla, David, Adelaido García-Andrés e Isaac Sánchez-Juárez (2015), "Identification of key productive sector in the Mexican economy", Expert Journal of Economics, 3(1), Expert Journals, Rumania, pp. 22-39.

Ros, Jaime (2015), ¿Cómo salir de la trampa del lento crecimiento y alta desigualdad? El Colegio de México y UNAM, Ciudad de México.

Sánchez, Isaac (2013), "Política industrial activa como estrategia para el crecimiento de la economía mexicana", Cuadernos de Trabajo de la UACJ en Estudios Regionales en Economía, Población y Desarrollo, 15, Universidad Autónoma de Ciudad Juárez, México, pp. 1-29.

Sánchez, Isaac y Juan C. Moreno-Brid (2016), "El reto del crecimiento eco- 
nómico en México: industrias manufactureras y política industrial", $\mathrm{Fi}$ nanzas y Política Económica, 8(2), Universidad Católica de Colombia, Colombia, pp. 271-299. DOI: http://dx.doi.org/10.14718/revfinanzpolitecon.2016.8.2.4

Sánchez-Juárez, Isaac y Rosa García-Almada (2016), "Public debt, public investment and economic growth in Mexico", International Journal of Financial Studies, 4(2), MDPI, Suiza, pp. 1-14. DOI: http://dx.doi. org/10.3390/ijfs4020006

Sánchez-Juárez, Isaac (2018), "Gasto público, índice de competitividad y política social en México", Problemas del Desarrollo, 49(192), Instituto de Investigaciones Económicas UNAM, México, pp. 109-138. DOI: https:// doi.org/10.22201/iiec.20078951e.2018.192.58918

Sánchez-Juárez, Isaac (2019), "Ciclos económicos en México: identificación, profundidad y duración", EconomíaUNAM, 16(47), Facultad de Economía de la UNAM, México, p. 93-108.

Srithongrung, Arwiphawee e Isaac Sánchez-Juárez (2015), "Fiscal policies and subnational economic growth in Mexico", International Journal of Economics and Financial Issues, 5(1), EconJournals, Turquía, pp. 11-22.

The Conference Board (2018), The conference board total economy database (Adjusted version), May 2017. Recuperado de https://www. conference-board.org/retrievefile.cfm?filename=TED_1_MAY20171. $\mathrm{xls} \times \&$ type $=$ subsite

Trejo, Alejandra (2017), "Crecimiento económico e industrialización en la agenda 2030: perspectivas para México", Problemas del Desarrollo, 48(188), Instituto de Investigaciones Económicas UNAM, México, pp. 83-111.

Vargas, Gustavo y Albino Luna (2014), "Slow growth in the mexican economy", Journal of Post Keynesian Economics, 37(1), Taylor and Francis, Reino Unido, pp. 115-134. DOI: https://doi.org/10.2753/PKE01603477370108

Williamson, John (1998), "Revisión del consenso de Washington", en Lois Emmerij y José Nuñéz (comps.), El desarrollo económico y social en los umbrales del siglo XXI. Banco Interamericano de Desarrollo, Washington, pp. 51-65.

Yifu, Justin (2014), "The Washington consensus revisited: a new structural economics perspective", Journal of Economic Policy Reform, 18(2), Taylor and Francis, Reino Unido, pp. 96-113. DOI: https://doi.org/10.1080/ 17487870.2014.936439 